\title{
Three-dimensional reconstruction method for measuring the knee valgus angle of the femur in northern Chinese adults*
}

\author{
Tong LIU ${ }^{\dagger 1}$, Chen-yu WANG ${ }^{2}$, Jian-lin XIAO ${ }^{1}$, Lan-yu ZHU ${ }^{3}$, Xue-zhou LI ${ }^{4}$, Yan-guo QIN ${ }^{\dagger 4}$, Zhong-li GAO ${ }^{1}$ \\ ( ${ }^{1}$ Department of Orthopedics, China-Japan Union Hospital of Jilin University, Changchun 130033, China) \\ ( ${ }^{2}$ Norman Bethune Medical School, Jilin University, Changchun 130021, China) \\ $\left({ }^{3}\right.$ School of Nursing, Changchun University of Chinese Medicine, Changchun 130117, China) \\ $\left({ }^{4}\right.$ Department of Orthopedics, the Second Hospital of Jilin University, Changchun 130041, China) \\ †E-mail: liutong1986@gmail.com; qinyanguo@hotmail.com \\ Received Jan. 22, 2014; Revision accepted May 11, 2014; Crosschecked July 25, 2014
}

\begin{abstract}
The purpose of this study was to establish a method for measuring the knee valgus angle from the anatomical and mechanical axes on three-dimensional reconstruction imaging models, and to use this method for estimating an average knee valgus angle value for northern Chinese adults. Computed tomographic angiography data in DICOM format for 128 normal femurs from 64 adult subjects were chosen for analysis. After the femur images were subjected to three-dimensional reconstruction, the deepest point in the intercondylar notch (point $A$ ), the midpoint of the medullary cavity $20 \mathrm{~cm}$ above the knee-joint line (point B), and the landmark of the femoral head rotation center (point $\mathrm{C}$ ) were identified on each three-dimensional model. The knee valgus angle was defined as the angle enclosed by the distal femoral anatomical axis (line $A B$ ) and the femoral mechanical axis (line $A C$ ). The average (mean $\pm S D$ ) of knee valgus angle for the 128 femurs was $6.20^{\circ} \pm 1.20^{\circ}$ (range, $3.05^{\circ}$ to $10.64^{\circ}$ ). Significant positive correlations were found between the knee valgus angles of the right and left sides and between the knee valgus angle and age. During total knee arthroplasty, choosing a valgus cut angle of approximately $6^{\circ}$ may achieve a good result in reestablishing the natural mechanical alignment of the lower extremity for patients of northern Chinese ethnicity. Larger valgus cut angles should be chosen for older patients.
\end{abstract}

Key words: Knee valgus angle, Three-dimensional reconstruction femoral model, Total knee arthroplasty, Northern Chinese adults, Preoperative design

doi: 10.1631 jzus.B1400019

Document code: A

CLC number: R687.4

\section{Introduction}

Total knee arthroplasty (TKA) is regarded as an effective treatment approach for late-stage osteoarthritis and other deformities of the knee joint, and studies have provided evidence of its long-term success in treating severe knee diseases (Insall and Kelly, 1986; Ranawat, 1986; Buechel et al., 1989). However, TKA is associated with high rates of revision reoper-

\footnotetext{
${ }^{\ddagger}$ Corresponding author

" Project supported by the Norman Bethune B Program of Jilin University (No. 2012216) and the Science and Technology Development Program of Jilin Province (No. 20100750), China

(C) Zhejiang University and Springer-Verlag Berlin Heidelberg 2014
}

ation after the primary arthroplasty (Pabinger et al., 2013). According to some reports, abnormal varus or valgus alignment may lead to loosening of the implants (Oswald et al., 1993; Nagamine et al., 2004). Thus, a key to minimizing the TKA failure rate is to reestablish the normal mechanical alignment of the knee joint, while preventing any abnormal varus or valgus errors.

To individualize alignment for each patient, some surgeons prefer to obtain the valgus angle preoperatively from a full-length X-ray radiograph (Sheehy et al., 2011). However, several imaging errors can occur with this method: for example, the images may not be strictly frontal or sagittal, extension 
deficits may occur, or rotation may exist between the femur and tibia (Krackow et al., 1990; Koshino et al., 2002; Kawakami et al., 2004; Radtke et al., 2010). Other surgeons use a general valgus angle, determined through experience, as a preferred option. Insall (1984) described a classical way of placing the femoral component at a knee valgus angle (KVA) of $7^{\circ}$ relative to the anatomical axis of the femur and placing the tibial component perpendicular to the tibial shaft. However, this approach was developed on the basis of the anatomic structure of people of western ethnicity.

It remains unclear whether TKA surgical experiences developed for western populations are also suitable for use in Chinese patients. Although a KVA of $5^{\circ}$ was recently reported for people of southern Chinese ethnicity (Wang et al., 2010), because of the enormous population in China, more evidence should be obtained and specific methods for measuring the parameters of the lower extremities in people of northern Chinese ethnicity should be determined. Therefore, we sought to develop a method for measuring the KVA from the anatomical and mechanical axes on three-dimensional (3D) reconstruction models. We applied this method to estimate an average KVA value for northern Chinese adults.

\section{Materials and methods}

\subsection{Study subjects}

This retrospective study was approved by our internal review board, and the patients and/or their families were informed that data from this case study would be submitted for publication, and gave their consent. Between April 2012 and February 2013, computer tomographic angiography (CTA) scanning data of the lower extremities were obtained at the authors' hospital for 128 femurs of 64 subjects (38 males), all from northern China. All CTA scans were done for the purpose of diagnosing vascular disease. The average age of the 64 patients was 59.3 years (range, 34 to 69 years), average height was $163.2 \mathrm{~cm}$ (range, 145 to $185 \mathrm{~cm}$ ), and average weight was $64.7 \mathrm{~kg}$ (range, 48 to $87 \mathrm{~kg}$ ). Subjects were included if they did not have severe osteoarthritis change in the knee or any deformity in the femur. CTA scanning was performed using a Toshiba Aquilion CT scanner
(Toshiba Corp., Tokyo, Japan) with the patient in the supine position, with his or her knees fully extended. The slice thickness was $2.0 \mathrm{~mm}$, X-ray tube current was $50 \mathrm{~mA}$, and $\mathrm{KVP}$ was $120 \mathrm{kV}$.

\subsection{D reconstruction}

The CTA scanning images were saved in DICOM format and imported into the Mimics 10.01 software program, for use in producing a $3 \mathrm{D}$ reconstruction skeleton model. The first step towards achieving an integrated $3 \mathrm{D}$ reconstruction skeleton model was to set the threshold value. The segmentation object will contain only those pixels of the image with a value higher than or equal to the threshold value. In the present study, the minimal threshold values were adjusted to 180-210 (average, 195) to obtain proper femur outlines. After the threshold was complete, Calculate-3D was used to construct a new 3D model for the femur (Fig. 1a).

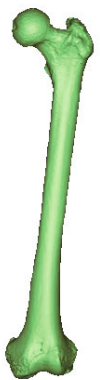

(a)

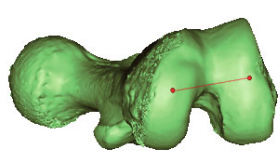

(b)

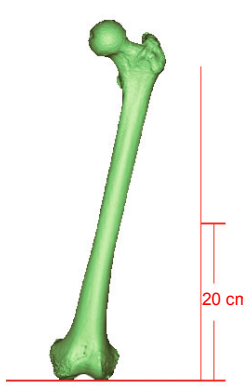

(c)
Fig. 1 Reconstructed femoral model

(a) Three-dimensional (3D) model of the femur; (b) Two centers are connected to form the knee-joint line; (c) The cross-section $20 \mathrm{~cm}$ above the knee-joint line was selected

\subsection{Locating landmarks}

First, the most distal cross-section containing the cortical bone of the intercondylar notch was selected. A circle tangent to the anteroposterior edges of the intercondylar notch cortical bone was created. The center of this circle was marked as point A, representing the deepest point in the intercondylar notch (Fig. 2). The most distal cross-sections of the medial and lateral condyles were selected, and close-fitting circles of the cortical bones of the condyles were created. The knee-joint line was created by connecting the two centers of the circles (Fig. 1b). A line perpendicular to the knee-joint line was made on the 
coronal section, and a point on the perpendicular line $20 \mathrm{~cm}$ above the intersection point was selected (Fig. 1c). Then, the corresponding cross-section was located. By closely fitting the medullary cavity with a circle, the landmark of the midpoint of the medullary cavity, point B, was obtained (Fig. 3).

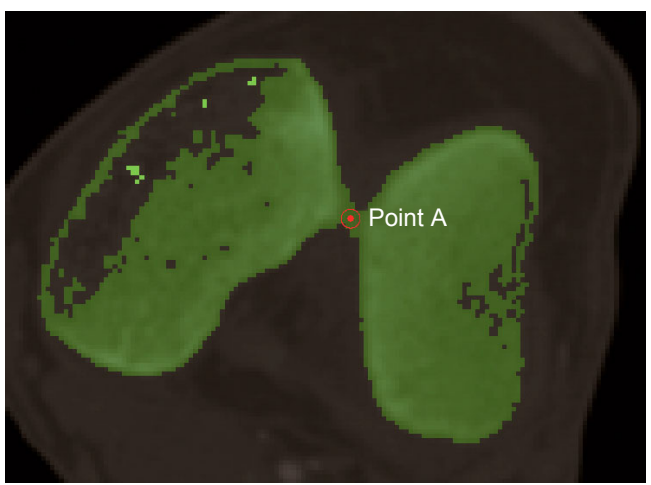

Fig. 2 Deepest point in the intercondylar notch (point A)

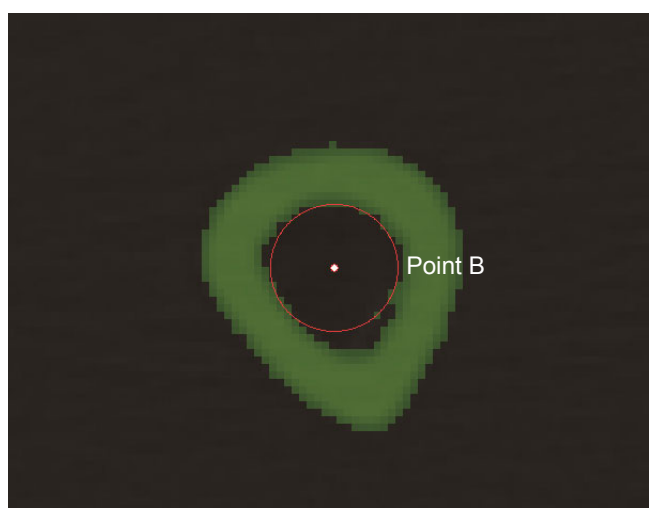

Fig. 3 Close fitting of the medullary cavity with a circle and the landmark of the midpoint (point $B$ )

The reconstructed 3D model was exported into a reverse-engineering software program (Geomagic Studio v12.0). The surface of the femoral head was selected. This surface was covered by cartilage and may contact the acetabulum. A sphere that closely fitted the selected area was created (Fig. 4), and the center served as the landmark of the femoral head rotation center (point $\mathrm{C}$ ). Because the close-fitting sphere only covers the maximum area with similar curvature, the fovea is automatically excluded from the sphere. Therefore, whether the fovea area is chosen in the fitting procedure or not will barely influence the result. In the present study, the fovea area was included to prevent any error due to manual editing.

\subsection{KVA formation}

To connect points $\mathrm{A}$ and $\mathrm{B}$, the line $\mathrm{AB}$ was formed as the distal femoral anatomical axis. To connect points $\mathrm{A}$ and $\mathrm{C}$, the line $\mathrm{AC}$ was formed as the mechanical axis of the femur. The KVA was defined as the angle enclosed by the distal anatomical axis $\mathrm{AB}$ and the mechanical axis AC (Fig. 5). Bilateral valgus angles were obtained from all 64 patients. To evaluate the statistical differences between genders and between sides, and to calculate the correlation between the KVA and age, height, weight, and body mass index (BMI), Student's $t$-test and Pearson's correlation coefficient were used in the PASW Statistics v18.0 software program. All of the above measurements were performed by one surgeon to eliminate interobserver bias.

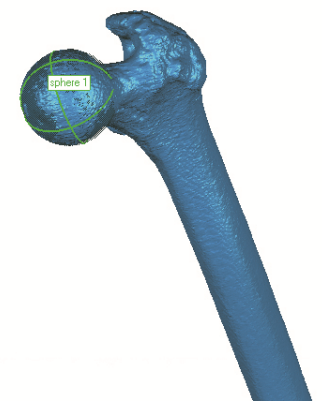

Fig. 4 Close fitting of the femoral head with a sphere

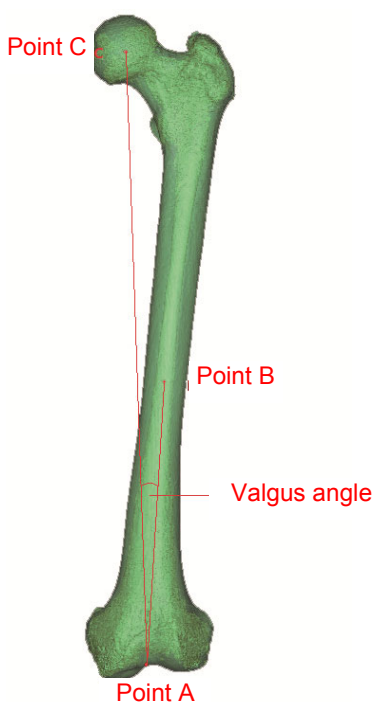

Fig. 5 Valgus angle enclosed by the distal anatomical axis $\mathrm{AB}$ and the mechanical axis $\mathrm{AC}$ 


\section{Results}

Results were divided into groups by gender and side (Table 1). The average KVA was $6.20^{\circ} \pm 1.20^{\circ}$ for all patients, $6.02^{\circ} \pm 1.25^{\circ}$ for male patients, $6.47^{\circ} \pm 1.08^{\circ}$ for female patients, $6.19^{\circ} \pm 1.31^{\circ}$ for the left side, and $6.21^{\circ} \pm 1.09^{\circ}$ for the right side. There was no significant difference $(P>0.05)$ between the KVAs of the left and right sides or between the KVAs of males and females in either side (Table 2). Positive correlations were obtained between the KVAs of the right and left sides $\left(R^{2}=0.649, P<0.01\right.$; Fig. 6a) and between the KVA and age $\left(R^{2}=0.079, P<0.01\right.$; Fig. 6b). There was no significant relationship between the KVA and the height, weight, or BMI.
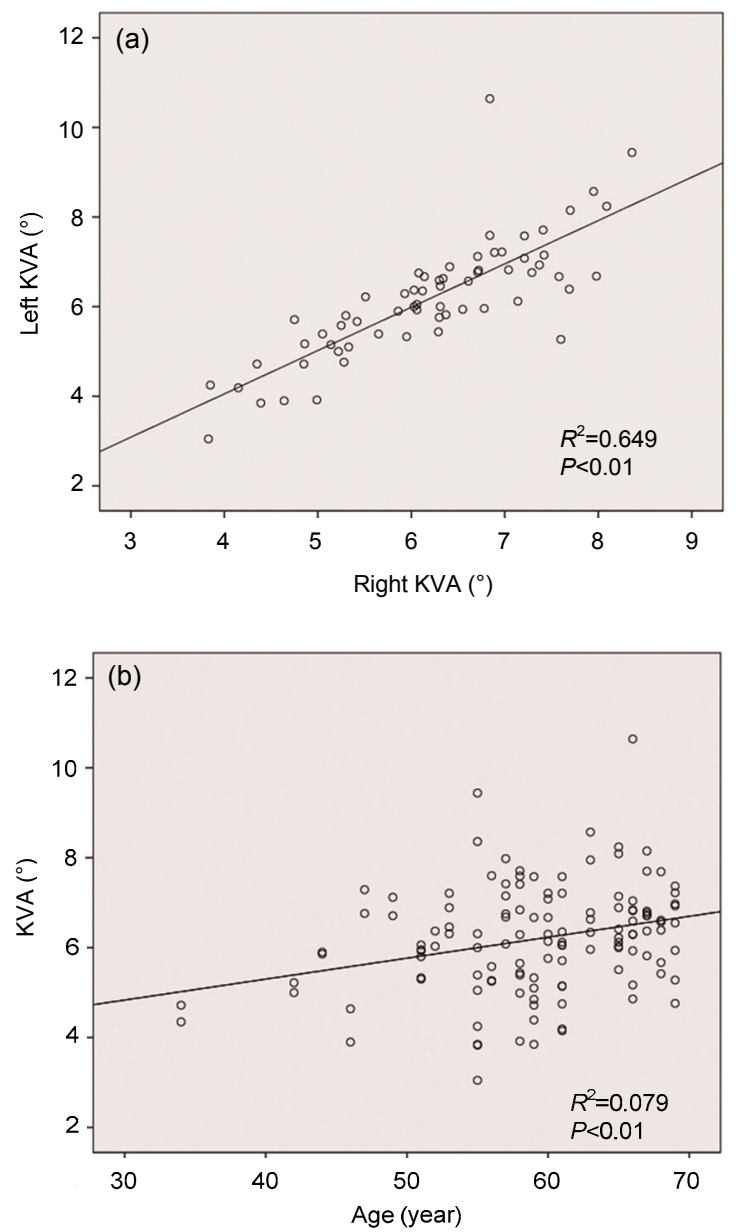

Fig. 6 Correlations between knee valgus angle (KVA) of the right and left sides (a) and between KVA and age (b)
Table 1 Knee valgus angle (KVA) data by gender and side

\begin{tabular}{|c|c|c|c|}
\hline \multirow{2}{*}{ Parameter } & \multirow{2}{*}{$n$} & \multicolumn{2}{|c|}{$\operatorname{KVA}\left(^{\circ}\right)$} \\
\hline & & Average $^{*}$ & Range \\
\hline Total & 128 & $6.20 \pm 1.20$ & $3.05-10.64$ \\
\hline Male & 76 & $6.02 \pm 1.25$ & $3.05-10.64$ \\
\hline Female & 52 & $6.47 \pm 1.08$ & $4.15-8.57$ \\
\hline Left & 64 & $6.19 \pm 1.31$ & $3.05-10.64$ \\
\hline Right & 64 & $6.21 \pm 1.09$ & $3.83-8.36$ \\
\hline Male-left & 38 & $6.04 \pm 1.44$ & $3.05-10.64$ \\
\hline Male-right & 38 & $6.00 \pm 1.06$ & $3.83-8.36$ \\
\hline Female-left & 26 & $6.42 \pm 1.10$ & $4.19-8.57$ \\
\hline Female-right & 26 & $6.52 \pm 1.09$ & $4.15-8.09$ \\
\hline
\end{tabular}

Table 2 Statistical analysis of knee valgus angle (KVA) by gender and side

\begin{tabular}{lcc}
\hline \multicolumn{1}{c}{ Parameter } & \multicolumn{1}{c}{$t$} & $P$ \\
\hline Left/right & -0.192 & 0.848 \\
Male/female & & \\
Right & -1.916 & 0.060 \\
Left & 0.297 & 0.257 \\
\hline
\end{tabular}

\section{Discussion}

\subsection{Methods of measuring the valgus angle}

Surgeons have developed various methods to obtain a generic KVA for TKA. Moreland et al. (1987) described two ways to form the valgus angle. These approaches have been considered as classical methods for obtaining the KVA. In both methods, two steps were applied. In the first step, femoral anatomic axis I was defined as a line connecting femoral shaft center I (located by bisecting the proximal-to-distal femur length and the mid-shaft medial-to-lateral femur width) with the center of the knee. Femoral shaft center II was located $10 \mathrm{~cm}$ above the center of the knee, midway between the medial and lateral surfaces, and femoral anatomic axis II was defined by a line drawn between femoral shaft center I and femoral shaft center II. In the second step, valgus angles I and II were separately formed by the intersection of the femoral anatomic axes I and II with the mechanical 
axis of the femur. Most studies of the lower extremity mechanical axis have been generally based on these two methods.

Oswald et al. (1993) developed a new method to achieve the KVA, which was developed from the theories of Moreland et al. (1987). They separately defined the distal femoral anatomical axis (connecting the midpoint of the cortical shaft $20 \mathrm{~cm}$ above the knee-joint line with the deepest point in the intercondylar notch), the central femoral anatomical axis (connecting the midpoint of the cortical shaft at one-half of the femoral length with the midpoint of the cortical shaft $10 \mathrm{~cm}$ above the joint line), and the long femoral anatomical axis (connecting the midpoint of the cortical shaft at two-thirds of the femoral length with the midpoint of the cortical shaft at one-third of the femoral length). Then, the angles a1, a2, and a3 were formed by the intersection of the mechanical axis with the distal, long, and central femoral anatomical axes. After this study was published, angle a1 was usually used as the standard valgus cut angle in preoperative designs by many surgeons.

In our study, we applied the distal femoral anatomical axis introduced by Oswald et al. (1993) rather than the classical anatomical axis to simulate the intramedullary guiding rod used in TKA. Another advantage of applying this axis is to reduce affection of femoral bowing on axial alignment (Lasam et al., 2013). The definition of the proximal point of the distal femoral anatomical axis was altered slightly. In particular, we replaced the midpoint of the femur outer shaft by the midpoint of the femoral medullary cavity $20 \mathrm{~cm}$ above the knee-joint line to reestablish the actual situation in the TKA operation. At the same time, in accordance with Maquet (1980) and Kapandji (1964), the deepest point of the intercondylar notch was chosen as the center of the knee. This point is an important landmark in the TKA operation, which can be observed easily in the preoperative design and during the operation.

We also made several improvements in our 3D reconstruction method. A reverse-engineering software program (Geomagic Studio 12) was used to distinguish the geometric characteristics on the reconstructed model and to achieve an appropriately close fitting to obtain accurate 3D coordinates. Each point was obtained from the models through a method of geometric close fitting.

\subsection{Evaluating the results}

The average KVA of the patients was $6.20^{\circ} \pm$ $1.20^{\circ}$. This result is sufficient evidence that the KVA for northern Chinese adults can be estimated at around $6.2^{\circ}$, which is very close to the $6^{\circ}$ suggested by Oswald et al. (1993) and Deakin et al. (2012), but it is a little smaller than the classic $7^{\circ}$ proposed by Insall (1984). The closeness of our value to that of Oswald et al. (1993) is probably because our method was based on his method. Compared with the KVA of $5^{\circ}$ suggested by Wang et al. (2010), our result was much closer to more commonly preferred values. Our finding suggests that surgeons who work in northern China and choose a valgus cut angle of around $6^{\circ}$ may achieve a good result for reestablishing the natural mechanical alignment of the lower extremity in most cases.

According to the statistical analysis, KVA showed a significant positive correlation with age, implying that the KVA increased as people aged. Research has shown that the geometry of the femur can change with aging (Crabtree et al., 2000; Riggs et al., 2004). Our findings provide new evidence to support these theories. However, since the amount of change in KVA due to age is very small $f(x)=$ $0.047 x+3.439$ ), a smaller or larger valgus cut angle will be only needed for very young or very old patients.

The KVAs of the left and right sides of the patients showed a positive correlation, confirming that the anatomical structure is bilaterally similar in an individual. However, the valgus angle was not related to BMI, height, or weight among patients of northern Chinese ethnicity. Some surgeons tend to use a larger valgus cut angle when a patient is very short and decrease the cut angle when the patient's height is much higher than average. Our result suggests that this trend should not be used among patients of northern Chinese ethnicity. In this study, KVAs ranged from $3.05^{\circ}$ to $10.64^{\circ}$, suggesting that setting the valgus cut angle at around $6^{\circ}$ may elicit valgus or varus mechanical alignments for patients who have extremely small or large valgus angles. Preoperative design must be done for the few patients with such 
anatomical variations. Deakin and Sarungi (2014) also supported this conclusion.

\subsection{Limitations}

We used data obtained from CTA images, and the incidence of vascular disease (and, thus, the need for CTA) in young people is generally low. Therefore, the number of young patients who were included in this study was limited. In this study, we only included the subjects without any severe osteoarthritis change in the knee joint. For those patients who have late-stage knee osteoarthritis with extreme varus or valgus deformity, our result of average KVA may no longer be a good choice for their knee replacement surgeries.

\section{Compliance with ethics guidelines}

Tong LIU, Chen-yu WANG, Jian-lin XIAO, Lan-yu ZHU, Xue-zhou LI, Yan-guo QIN, and Zhong-li GAO declare that they have no conflict of interest.

All procedures followed were in accordance with the ethical standards of the responsible committee on human experimentation (institutional and national) and with the Helsinki Declaration of 1975, as revised in 2008 (5). Informed consent was obtained from all patients for being included in the study.

\section{References}

Buechel, F.F., Rosa, R.A., Pappas, M.J., 1989. A metal-backed, rotating-bearing patellar prosthesis to lower contact stress: an 11-year clinical study. Clin. Orthop. Relat. Res., (248): 34-49. [doi:10.1097/00003086-198911000-00008]

Crabtree, N., Lunt, M., Holt, G., et al., 2000. Hip geometry, bone mineral distribution, and bone strength in European men and women: the EPOS study. Bone, 27(1):151-159. [doi:10.1016/S8756-3282(00)00300-8]

Deakin, A.H., Sarungi, M., 2014. A comparison of variable angle versus fixed angle distal femoral resection in primary total knee arthroplasty. J. Arthroplasty, 29(6): 1133-1137. [doi:10.1016/j.arth.2013.11.009]

Deakin, A.H., Basanagoudar, P.L., Nunag, P., et al., 2012. Natural distribution of the femoral mechanical-anatomical angle in an osteoarthritic population and its relevance to total knee arthroplasty. Knee, 19(2):120-123. [doi:10. 1016/j.knee.2011.02.001]

Insall, J.N., 1984. Surgery of the Knee. Churchill Livingstone, New York, p.828.

Insall, J.N., Kelly, M., 1986. The total condylar prosthesis. Clin. Orthop. Relat. Res., (205):43-48. [doi:10.1097/ 00003086-198604000-00006]

Kapandji, I.A., 1964. Illustrated physiology of joints. Med.
Biol. Illus., 14:72-81.

Kawakami, H., Sugano, N., Yonenobu, K., et al., 2004. Effects of rotation on measurement of lower limb alignment for knee osteotomy. J. Orthop. Res., 22(6):1248-1253. [doi:10. 1016/j.orthres.2004.03.016]

Koshino, T., Takeyama, M., Jiang, L.S., et al., 2002. Underestimation of varus angulation in knees with flexion deformity. Knee, 9(4):275-279. [doi:10.1016/S0968-0160 (02)00049-2]

Krackow, K.A., Pepe, C.L., Galloway, E.J., 1990. A mathematical analysis of the effect of flexion and rotation on apparent varus/valgus alignment at the knee. Orthopedics, 13(8):861-868.

Lasam, M.P., Lee, K.J., Chang, C.B., et al., 2013. Femoral lateral bowing and varus condylar orientation are prevalent and affect axial alignment of TKA in Koreans. Clin. Orthop. Relat. Res., 471(5):1472-1483. [doi:10.1007/ s11999-012-2618-7]

Maquet, P., 1980. The biomechanics of the knee and surgical possibilities of healing osteoarthritic knee joints. Clin. Orthop. Relat. Res., 146:102-110. [doi:10.1097/0000 3086-198001000-00014]

Moreland, J.R., Bassett, L.W., Hanker, G.J., 1987. Radiographic analysis of the axial alignment of the lower extremity. J. Bone Joint. Surg. Am., 69(5):745-749.

Nagamine, R., Kondo, K., Ikemura, S., et al., 2004. Distal femoral cut perpendicular to the mechanical axis may induce varus instability in flexion in medial osteoarthritic knees with varus deformity in total knee arthroplasty: a pitfall of the navigation system. J. Orthop. Sci., 9(6): 555-559. [doi:10.1007/s00776-004-0818-z]

Oswald, M.H., Jakob, R.P., Schneider, E., et al., 1993. Radiological analysis of normal axial alignment of femur and tibia in view of total knee arthroplasty. J. Arthroplasty, 8(4):419-426. [doi:10.1016/S0883-5403(06)80042-2]

Pabinger, C., Berghold, A., Boehler, N., et al., 2013. Revision rates after knee replacement. Cumulative results from worldwide clinical studies versus joint registers. Osteoarthritis Cartilage, 21(2):263-268. [doi:10.1016/j. joca.2012.11.014]

Radtke, K., Becher, C., Noll, Y., et al., 2010. Effect of limb rotation on radiographic alignment in total knee arthroplasties. Arch. Orthop. Trauma Surg., 130(4):451-457. [doi:10.1007/s00402-009-0999-1]

Ranawat, C.S., 1986. The patellofemoral joint in total condylar knee arthroplasty. Pros and cons based on five- to ten-year follow-up observations. Clin. Orthop. Relat. Res., (205):93-99. [doi:10.1097/00003086-198604000-00012]

Riggs, B.L., Melton III, L.J., Robb, R.A., et al., 2004. Population-based study of age and sex differences in bone volumetric density, size, geometry, and structure at different skeletal sites. J. Bone Miner. Res., 19(12): 1945-1954. [doi:10.1359/jbmr.040916] 
Sheehy, L., Felson, D., Zhang, Y., et al., 2011. Does measurement of the anatomic axis consistently predict hipknee-ankle angle (HKA) for knee alignment studies in osteoarthritis? Analysis of long limb radiographs from the multicenter osteoarthritis (MOST) study. Osteoarthritis Cartilage, 19(1):58-64. [doi:10.1016/j.joca.2010.09.011]
Wang, Y., Zeng, Y., Dai, K., et al., 2010. Normal lowerextremity alignment parameters in healthy Southern Chinese adults as a guide in total knee arthroplasty. $J$. Arthroplasty, 25(4):563-570. [doi:10.1016/j.arth.2009. 03.021]

\section{中文㔍要:}

\section{本文题目：三维重建法测量中国北方成人股骨膝外翻角}

Three-dimensional reconstruction method for measuring the knee valgus angle of the femur in northern Chinese adults

研究目的: 利用三维重建影像模型获取膝外翻角, 并通过使用这种方法来测量中国北方成人膝外翻角 的平均估计值。

创新要点: 本研究所获取的膝外翻角平均值对于在中国北方人群进行膝关节置换术时有着重要的参考 意义，新的三维重建测量法使得膝外翻角的术前个体化测量更加精确。

研究方法: 利用 64 名患者的 CT 血管造影 (CTA) 的 DICOM 数据获取共 128 件股骨三维重建模型。 在重建的股骨模型上分别定位髁间凹最高点（点 A）、膝关节线上 $20 \mathrm{~cm}$ 截面的髓腔中点 （点 B）和股骨头旋转中心（点 C)。之后膝关节外翻角即由股骨远端解剖轴（线 AB）与 股骨机械轴（线 AC）所围成（上述定位过程详见图 5)。经统计分析后，128 件股骨的平 均外翻角值为 $6.20^{\circ} \pm 1.20^{\circ}$ 。统计分析还发现, 膝外翻角与个体年龄呈显著性正相关, 同一 个体的左右侧外翻角呈显著性正相关。

重要结论: 在对北方成年人群进行膝关节置换术时, 选取外翻角为 $6^{\circ}$ 可会在重建人体下肢力线上获得 更好的术后效果。在对年长患者进行手术时应选用更大的外翻角。

关键词组：膝外翻角；股骨三维重建模型；中国北方成人；膝关节置换术；术前设计 